\title{
Multiple Evanescent White Dot Syndrome after Human Papillomavirus Vaccination
}

\author{
Ken Ogino ${ }^{a}$ Shoji Kishi ${ }^{b} \quad$ Nagahisa Yoshimura ${ }^{a}$ \\ ${ }^{a}$ Department of Ophthalmology and Visual Sciences, Kyoto University Graduate School \\ of Medicine, Kyoto, and 'bepartment of Ophthalmology, Gunma University School of \\ Medicine, Maebashi, Japan
}

\section{Key Words}

Multiple evanescent white dot syndrome $\cdot$ Acute zonal occult outer retinopathy · Human papillomavirus · Vaccination

\begin{abstract}
Although the cause of multiple evanescent white dot syndrome (MEWDS) has not been elucidated, 2 reports have described cases of MEWDS after vaccination. A 16-year-old girl presented with throat pain, headache, and photopsia in the left eye 2 weeks after receiving a human papillomavirus (HPV) vaccination. The clinical examination revealed numerous white dots on the fundus, visual field loss, and abnormal electroretinography results, which are concordant with a diagnosis of MEWDS. Although the white dots on the fundus disappeared within a few months, her visual field continued to deteriorate progressively over 1.5 years of follow-up. Images obtained by fundus autofluorescence and optical coherence tomography revealed longitudinal changes consistent with the observed functional losses. Immunosuppressive therapy (betamethasone $200 \mathrm{mg} /$ day) resulted in the complete resolution of all fluorescein leakage from the retinal vasculature. Maintenance betamethasone treatment $(0.5$ $\mathrm{mg} /$ day) led to visual field results over a period of 6 months. In this case of MEWDS, subsequent to HPV vaccination, the results of fluorescein angiography showed a marked response to immunosuppressive therapy. These findings support the hypothesis that MEWDS is associated with an impaired immune function.

(C) 2014 S. Karger AG, Basel
\end{abstract}


Ogino et al.: Multiple Evanescent White Dot Syndrome after Human Papillomavirus Vaccination

\section{Introduction}

Multiple evanescent white dot syndrome (MEWDS), first reported by Jampol et al. [1] in 1984 , is an acute retinopathy that typically manifests unilaterally in young adults. Although numerous studies have characterized the associated clinical features based on the results of fluorescein and indocyanine green angiography [1, 2], electroretinography (ERG) [3], and optical coherence tomography (OCT) [4], the cause of this condition remains to be elucidated. Most patients complain of a sudden onset of vision loss, associated with photopsia and a preceding flu-like illness. Gass [5] proposed the possibility of a viral infection while Jampol et al. [6] suggested that MEWDS is a specific type of autoimmune disease.

MEWDS is usually a self-limiting condition with patients recovering and achieving a good visual acuity within several weeks, but some cases lead to acute zonal occult outer retinopathy (AZOOR) [7]. The prognosis may be difficult to determine due to the overlying similarities of MEWDS, multifocal choroiditis with panuveitis, punctate inner choroidopathy and AZOOR, all of which can involve white dots and/or an enlarged blind spot [8,9].

Here we report a case of MEWDS after a human papillomavirus (HPV) vaccination, showing progressive concentric visual field loss. The results of fluorescein angiography (FA) showed a marked response to immunosuppressive therapy.

\section{Case Reports}

At the end of August 2011, a 16-year-old girl presented to Gunma University Hospital with throat pain, headache, and photopsia in the left eye. She had received her second HPV vaccination (Cervarix ${ }^{\circledR}$, Glaxo Smith Kline) on August 10, 2011 (the first was on July 9, 2011 and the third on January 30,2012 ). The patient had also presented asymptomatically with high levels of anti-nuclear antibodies when she was 12 years old.

The patient's Snellen visual acuity was 1.2 in both eyes. Numerous white dots were seen in the nasal retina of the left eye (fig. 1a). Static perimetry showed sensitivity loss in the temporal inferior visual field of the left eye (fig. 1d). Full-field ERG was performed using the International Society for Clinical Electrophysiology (ISCEV) standard protocol. The results for all protocols revealed non-specific reduced amplitudes. OCT showed the disappearance of the inner-segment/outer-segment (IS/OS) line at the nasal retina (fig. 1i) and the intact fovea (fig. 1j). The diagnosis was MEWDS.

Two months later, the white dots had largely disappeared, but perimetry showed a worsening of the visual field in the left eye. Four months later, perimetry revealed further expansion of this loss of sensitivity in the superior nasal as well as inferior temporal fields (fig. 1e).

Seven months later, the patient visited Kyoto University Hospital in search of a second opinion. Her visual acuity was 1.2 in both eyes. Arterial narrowing was observed (fig. 1c), but no white dot or bone spicule pigmentation was visible. OCT revealed a parafoveal photoreceptor loss (fig. 1k). Areas of hyper-autofluorescence were observed at the posterior pole in the left eye (fig. 2c). FA revealed leakage from the mid-peripheral vasculature (fig. 2e). ISCEV standard ERG showed subnormal amplitudes and the patient's laboratory results were negative for anti-recoverin antibody; no other specific retinal antibody was detected by Western blotting in our laboratory. The patient's atypical scotoma and the leakage observed on FA were not compatible with AZOOR while the lack of retina-specific antibodies ruled out autoimmune retinopathy. 
One and a half years after the onset of symptoms, the patient reported a subjective worsening of her visual field. The perimetry results supported the diagnosis of a scotoma enlargement (fig. 1g). The areas of hyper-autofluorescence at the posterior pole showed further concentric restrictions (fig. 2d). FA was performed again and revealed a marked increase in vascular leakage (fig. 2f). Notably, the administration of fluorescein induced nausea and a skin rash that resolved rapidly. We then initiated immunosuppressive therapy (methylprednisolone 1,000 mg/day), after which the patient reported throat discomfort and a return of the skin rash. We stopped the use of prednisolone because of this allergic response. Instead, the patient was treated with betamethasone $(200 \mathrm{mg}$ ) and anti-histamine for 3 days. The results of FA performed 1 week after therapy showed a drastic reduction in leakage from the vasculature (fig. $2 \mathrm{~g}$ ). The course of steroid treatment was tapered gradually to a maintenance dose of $0.5 \mathrm{mg}$ betamethasone/day. Six months after the initiation of this course of treatment, the perimetry results showed no further regression of the patient's visual field (fig. 1h). However, FA revealed recurrent leakage (fig. 2h).

\section{Discussion}

The patient's initial symptoms were characteristic of MEWDS: flu-like illness, photopsia, numerous yellow-white spots at the level of the retinal pigment epithelium, and abnormal ERG results. Her visual field continued to worsen for 2 years after the white dots on her fundus had disappeared. Exacerbation of the patient's symptoms was paralleled by a decrease in size of the area of hyper-autofluorescence as well as a shortening of the IS/OS line. These symptoms are not typically associated with MEWDS. So far, recurrence [10], AZOOR [7], and/or choroidal neovascularization [11] have been reported after MEWDS, and it might be possible to diagnose this case as AZOOR with MEWDS depending on the abnormal thinning of the outer retina. However, we noticed differences compared to previous cases of AZOOR associated with MEWDS, which had a unique history of HPV vaccination and marked leakage from the vasculature in FA.

Prophylactic vaccines against HPV represent a cost-effective tool which can reduce the incidence of cervical cancer. Cervarix, which is a bivalent vaccine against HPV types 16 and 18, was approved in 2009. The GlaxoSmithKline Vaccine HPV-007 Study Group concluded that the frequency of serious adverse events in the vaccine group was similar to that observed in the placebo group [12]. Myalgia, fatigue, headache, cold, rash, and fever are known as mild and common adverse events, but there has been no report of eye disease secondary to HPV vaccination. However, the occurrence of MEWDS was previously reported in association with hepatitis A and B $[13,14]$. Considering the patient's recent vaccination, her history of abnormal antinuclear antibody levels, and her allergic response to several drugs, the HPV vaccination may have induced her abnormal retinal findings.

To our knowledge, the previously reported case of AZOOR accompanied by MEWDS showed minimal leakage on FA [15], but in our case, leakage was present at 7 months and increased at 17 months after onset. Generally, vascular leakage indicates a breakdown of the blood-retinal barrier. In diabetic retinopathy or retinal vein occlusion, elevated levels of vascular endothelial growth factor (VEGF) increase vascular hyperpermeability. The standard approach to therapy is anti-VEGF therapy. In uveitis, an autoimmune eye disease, phlebitis is usually controlled by immunosuppressive drugs such as steroids. In this case, the patient's serum samples showed no evidence of retina-specific autoantibodies. Her worsening vision urged us to start immunosuppressive therapy along with autoimmune retinopathy, and that resulted spontaneously in a marked improvement of leakage. Five 
Ogino et al.: Multiple Evanescent White Dot Syndrome after Human Papillomavirus vaccination

months after steroid pulse therapy, on a maintenance dose of $0.5 \mathrm{mg} /$ day, recurring leakage was observed. Due to the short-term nature of our observation period, we could not determine whether our approach to management will ultimately preserve the patient's visual function. However, her rapid response to steroid treatment indicates that the pathogenesis of her condition involved an impaired autoimmune response.

This case of MEWDS after HPV vaccination showed progressive concentric visual field loss. FA revealed a dramatic response to immunosuppressive therapy. This supports the hypothesis that the cause of MEWDS is related to immune function.

\section{References}

1 Jampol LM, Sieving PA, Pugh D, Fishman GA, Gilbert H: Multiple evanescent white dot syndrome. 1. Clinical findings. Arch Ophthalmol 1984;102:671-674.

2 Ie D, Glaser BM, Murphy RP, Gordon LW, Sjaarda RN, Thompson JT: Indocyanine green angiography in multiple evanescent white-dot syndrome. Am J Ophthalmol 1994;117:7-12.

3 Sieving PA, Fishman GA, Jampol LM, Pugh D: Multiple evanescent white dot syndrome. 2. Electrophysiology of the photoreceptors during retinal pigment epithelial disease. Arch Ophthalmol 1984;102:675-679.

4 Li D, Kishi S: Restored photoreceptor outer segment damage in multiple evanescent white dot syndrome. Ophthalmology 2009;116:762-770.

5 Gass JD: Are acute zonal occult outer retinopathy and the white spot syndromes (AZOOR complex) specific autoimmune diseases? Am J Ophthalmol 2003;135:380-381.

6 Jampol LM, Becker KG: White spot syndromes of the retina: a hypothesis based on the common genetic hypothesis of autoimmune/inflammatory disease. Am J Ophthalmol 2003;135:376-379.

7 Gass JD: Acute zonal occult outer retinopathy. Donders Lecture: The Netherlands Ophthalmological Society, Maastricht, Holland, June 19, 1992. J Clin Neuroophthalmol 1993;13:79-97.

-8 Reddy CV, Brown J Jr, Folk JC, Kimura AE, Gupta S, Walker J: Enlarged blind spots in chorioretinal inflammatory disorders. Ophthalmology 1996;103:606-617.

-9 Callanan D, Gass JD: Multifocal choroiditis and choroidal neovascularization associated with the multiple evanescent white dot and acute idiopathic blind spot enlargement syndrome. Ophthalmology 1992;99:1678-1685.

10 Oh KT, Christmas NJ, Russell SR: Late recurrence and choroidal neovascularization in multiple evanescent white dot syndrome. Retina 2001;21:182-184.

$\checkmark 11$ Rouvas AA, Ladas ID, Papakostas TD, Moschos MM, Vergados I: Intravitreal ranibizumab in a patient with choroidal neovascularization secondary to multiple evanescent white dot syndrome. Eur J Ophthalmol 2007;17:996-999.

12 GlaxoSmithKline Vaccine HPVSG, Romanowski B, de Borba PC, Naud PS, Roteli-Martins CM, De Carvalho NS, Teixeira JC, Aoki F, Ramjattan B, Shier RM, Somani R, Barbier S, Blatter MM, Chambers C, Ferris D, Gall SA, Guerra FA, Harper DM, Hedrick JA, Henry DC, Korn AP, Kroll R, Moscicki AB, Rosenfeld WD, Sullivan BJ, Thoming CS, Tyring SK, Wheeler CM, Dubin G, Schuind A, Zahaf T, Greenacre M, Sgriobhadair A: Sustained efficacy and immunogenicity of the human papillomavirus (HPV)-16/18 AS04-adjuvanted vaccine: analysis of a randomised placebo-controlled trial up to 6.4 years. Lancet 2009;374:1975-1985.

13 Baglivo E, Safran AB, Borruat FX: Multiple evanescent white dot syndrome after hepatitis B vaccine. Am J Ophthalmol 1996;122:431-432.

14 Stangos A, Zaninetti M, Petropoulos I, Baglivo E, Pournaras C: Multiple evanescent white dot syndrome following simultaneous hepatitis-A and yellow fever vaccination. Ocul Immunol Inflamm 2006;14:301-304.

15 Fine H, Spaide R, Ryan E, Matsumoto Y, Yannuzzi L: Acute zonal occult outer retinopathy in patients with multiple evanescent white dot syndrome. Arch Ophthalmol 2009;127:66-70. 


\section{Case Reports in \\ Ophthalmology}

\begin{tabular}{l|l}
\hline Case Rep Ophthalmol 2014;5:38-43 & \\
\hline DOI: 10.1159/000358870 & $\begin{array}{l}\text { ○ 2014 S. Karger AG, Basel } \\
\text { www.karger.com/cop }\end{array}$ \\
\hline
\end{tabular}

Ogino et al.: Multiple Evanescent White Dot Syndrome after Human Papillomavirus Vaccination

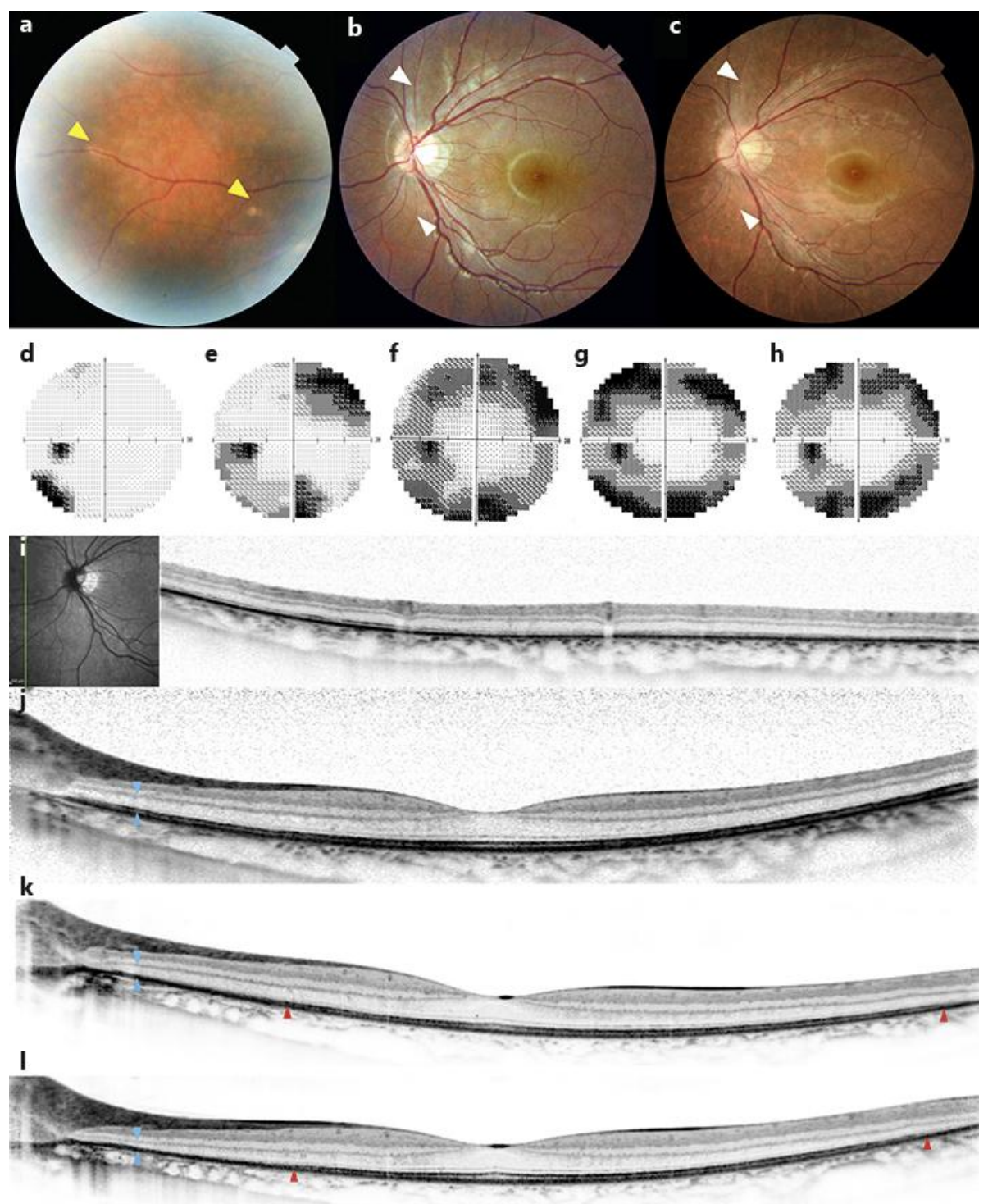

Fig. 1. Color fundus photograph obtained at the first visit $(\mathbf{a}, \mathbf{b})$ and 2 years later (c). Yellow arrowheads indicate the white dots in the nasal retina. White arrowheads indicate longitudinal narrowing of the retinal arteries. Visual fields measured using the 30-2 SITA standard program on the Humphrey Field Analyzer at baseline (d), 4 months (e), 1 year (f), 1.5 years (g), and 2 years (h). OCT images obtained at baseline (i, j), 7 months (k), and 1.5 years (I). The junction of the inner and outer segments (IS/OS) is difficult to discern in nasal retina (i), but normal surrounding the fovea (j). Blue and red arrowheads indicate a thinning of the outer retina and a shortening of the IS/OS, respectively. 


\section{Case Reports in Ophthalmology}

\begin{tabular}{l|l}
\hline Case Rep Ophthalmol 2014;5:38-43 \\
\hline DOI: 10.1159/000358870 & $\begin{array}{l}\text { ○ 2014 S. Karger AG, Basel } \\
\text { www.karger.com/cop }\end{array}$ \\
\hline
\end{tabular}

Ogino et al:: Multiple Evanescent White Dot Syndrome after Human Papillomavirus Vaccination

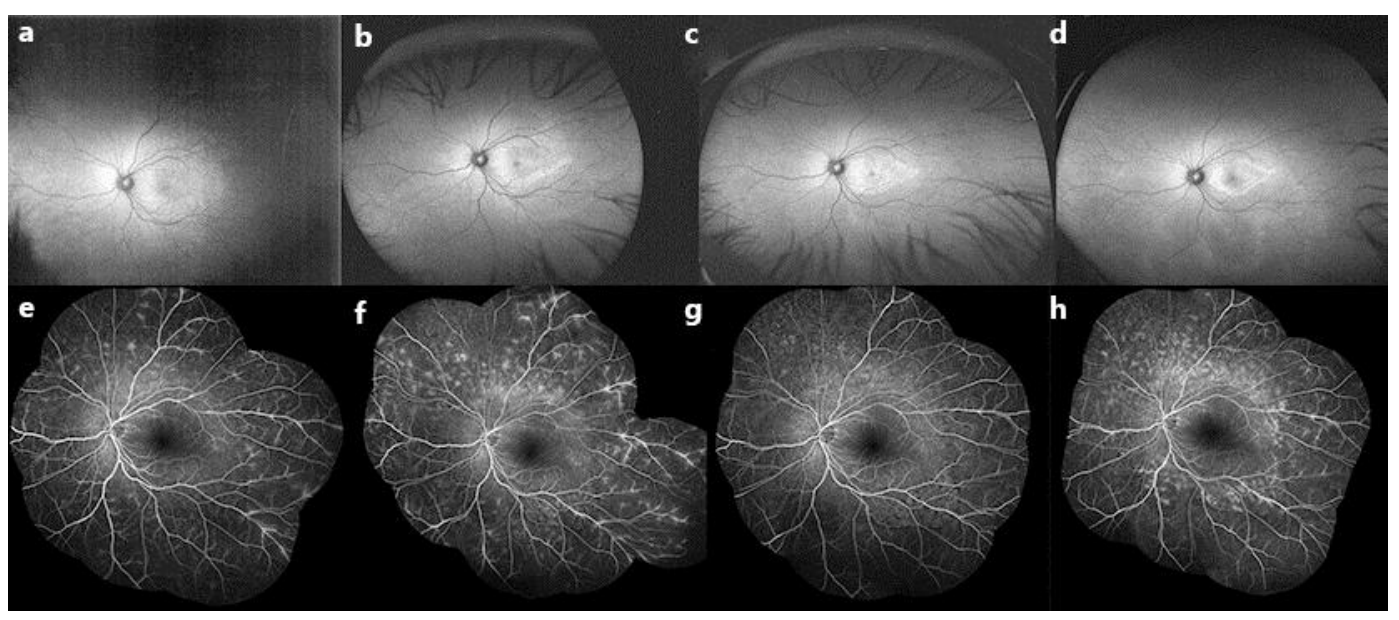

Fig. 2. Wide-field autofluorescence images at the first visit (a), 4 months (b), 7 months (c), and 1.5 years (d). Areas of hyper-autofluorescence were scattered mainly in the nasal retina at baseline (a) and gradually encroached upon the posterior pole (b-d). FA at 7 months (e), 1.5 years (f), 1 week after steroid pulse therapy (g), and after 2 years (h). Fluorescein leakage from the vasculature has resolved almost completely after therapy $(\mathrm{g})$. 